\title{
sciendo
}

\section{Training Loads and RSA and Aerobic Performance Changes During the Preseason in Youth Soccer Squads}

\author{
by \\ Tiago Cetolin 1,2, Anderson Santiago Teixeira1,2, Almir Schmitt Netto ${ }^{3}$, \\ Alessandro Haupenthal ${ }^{7}$, Fábio Yuzo Nakamura ${ }^{4,5,6}$, \\ Luiz Guilherme Antonacci Guglielmo², Juliano Fernandes da Silva ${ }^{1}$
}

\begin{abstract}
The aims of this study were to compare the internal training load (ITL) in soccer players of two competitive age groups (under-15 [U-15] and under-19 [U-19]) during an 8-week preseason training period and compare the associated changes in physical performance measures. Eighteen $U-15$ and twelve $U-19$ players were monitored over an 8-week period during the preseason phase. The ITL was monitored using the session rating of perceived exertion (RPE) method. Before and after the preseason period, physical performance was assessed by best (RSAbest) and mean (RSAmean) times in a repeated sprint ability (RSA) test and peak velocity derived from the Carminatti test (PVT-CAR). Total weekly ITL increased with age (U-15: $13770 \pm 874 \mathrm{AU}$ vs. U-19: $33584 \pm 2506 \mathrm{AU} ; p<0.001)$. In addition, $U-19$ players perceived training sessions as heavier than $U-15$ players $(6.1 \pm 0.3$ vs. $5.3 \pm 0.3 \mathrm{AU}$, respectively; $p<0.001)$. After the preseason period, very likely to almost certainly positive changes were observed for all performance measures in both age groups. However, the U-15 group had possibly superior gains in RSAbest $(+1.40 \%, 90 \% C L-0.29$ to 3.05, with ES $=0.35)$ and likely higher effects in RSAmean $(+1.89 \%, 90 \%$ CL 0.04 to 3.70, with ES $=0.53)$ and $P V_{T-C A R}(+2.71 \%, 90 \% C L 0.35$ to 5.01 , with $E S=0.37)$ compared to the U-19 group. In conclusion, our findings demonstrate that the U-19 group accumulate higher total weekly ITLs than the U-15 group during the preseason phase due to longer and heavier training sessions. However, the U-15 group obtained superior gains in soccer-specific physical abilities while accumulating half the total ITLs during lighter training sessions.
\end{abstract}

Key words: field testing; physical adaptation; rating of perceived exertion; team sports; youth athletes.

\section{Introduction}

Long-term athlete development (LTAD) programs underline that preparation of youths to become successful adult elite athletes is a multifactorial process (Vaeyens et al., 2008). Briefly, the current LTAD model available offers an integrative approach to optimize the athletic development of youths throughout the childhood and adolescence (i.e., identifying when and why

\footnotetext{
1 - Research Group for Development of Football and Futsal, Sports Center, Federal University of Santa Catarina, Florianópolis - SC, Brazil.

2 - Physical Effort Laboratory, Sports Center, Federal University of Santa Catarina, Florianópolis - SC, Brazil.

3 - Health Sciences and Sports Center, University of the State of Santa Catarina, Florianópolis - SC, Brazil.

4 - Department of Medicine and Aging Sciences, University Gabriele d'Annunzio of Chieti-Pescara, Italy.

5 - Nucleus of High Performance in Sport, São Paulo, Brazil.

6 - The College of Healthcare Sciences, James Cook University, Queensland, Australia.

7 - Aging, Resources, Rheumatology Laboratory, Federal University of Santa Catarina, Araranguá - SC, Brazil.
} 
training of each motor ability should be emphasized), taking into account the interaction between growth, maturation and training practices (Lloyd and Oliver, 2012). During childhood and adolescence, coaches and strength and conditioning professionals focus on developing all physical abilities and sportsspecific technical-tactical skills, aiming to progressively prepare their players to cope with high demands of competition in adulthood (Ford et al., 2011). To achieve this goal, and respecting the maturational status of the athletes (Lloyd et al., 2014), a gradual and systematic progression of overall training loads placed on youth players is expected. In theory, this planned progression will allow them to attain optimal development of their physical abilities, avoiding negative consequences of excessive training and insufficient recovery (e.g., injury, illness, and overtraining) (Gabbett et al., 2014; Wrigley et al., 2012). Careful examination of the current literature reveals that this optimal loading is not always observed in young team sports athletes (Noon et al., 2015; Oliver et al., 2015; Czuba et al., 2014).

Several studies have been designed to describe and quantify the training loads experienced during the preseason and in-season phases in junior (late adolescence) and senior players (Coutinho et al., 2015; Malone et al., 2015; Miloski et al., 2015; Rabelo et al., 2016). However, in the context of talent identification and development, it is widely advocated that the two main composite factors of the overall training load (i.e., volume and intensity), should be structured according to the biological development of the athlete (Ford et al., 2011; Gabbett et al., 2014). Although appealing, few studies have extensively addressed the training load profile in young players, with a special focus on comparing age groups over a longer period ( $>4-6$ weeks) during the team sports preseason (Gabbett et al., 2014). Objective data using GPS technology have suggested the existence of age- and maturityrelated variations in running match performance (e.g., total distance covered) (Buchheit et al., 2010c; Buchheit and Mendez-Villanueva 2014) and time-motion during technical-tactical training sessions (Abade et al., 2014; Gastin et al., 2013). In general, these studies support the notion that match and training demands (external load indicators) increase as players become older.
On the other hand, there are other studies showing that matches and training intensities, evaluated by internal load measures such as the rating of perceived exertion (RPE) or heart rate (HR), do not change across competitive age categories (e.g., U-14, U-16 and U-18) (MendezVillanueva et al., 2013; Wrigley et al., 2012). Of interest, several of these previous studies have been performed during the in-season phase, a period characterized by reduced training loads (volume and intensity) compared to the preseason phase (Malone et al., 2015; Miloski et al., 2015). In this sense, further studies during the preseason phase are required to provide additional information on training volume and intensity placed on adolescent players involved in systematic and standardized training programs.

The development of aerobic performance (i.e., intermittent endurance running capacity) and repeated sprint ability (RSA) during the specialization years in soccer is considered fundamental to compete at professional level (Roescher et al., 2010; Valente-dos-Santos et al., 2012). Intermittent endurance running and RSA are positively associated with the ability to perform intermittent high-intensity efforts during soccer matches (Da Silva et al., 2016; Rampinini et al., 2007). Furthermore, both have been successful in discriminating players of different competitive levels (Impellizzeri et al., 2008; Vaeyens et al., 2006). These data highlight the importance of well-designed preseason training to enhance intermittent endurance running and RSA performance. Some studies have reported positive gains in RSA and intermittent endurance running performance after controlled experimental interventions using different types of training (Buchheit et al., 2008, 2010a; Da Silva et al., 2015). However, further studies describing training loads and their subsequent effects on soccer match-related physical fitness attributes (i.e., intermittent endurance and RSA performances) in youth players after a regular and systematic training process without any external manipulation during the preseason phase are warranted. For technical staff dealing with adolescent players of different ages, it is also of practical interest and relevant to know whether the magnitude of adaptations in performance could be affected by age (Philippaerts et al., 2006). In practical terms, the resulting findings of this 
study can provide coaches and technical staffs with a better understanding of the player's adaptive capacity within each age group to enhance both intermittent endurance and RSA performance. In addition, this study may give some support in identifying a period of optimal trainability of intermittent endurance running capacity and RSA based on chronological age.

Thus, the aims of the present study were: (1) to compare internal training loads (as measured by the RPE) in soccer players of two competitive age groups (under-15 [U-15] and under-19 [U-19]) regularly training in a Brazilian professional soccer academy during the preseason phase (8-weeks), and (2) to compare the associated changes in physical performance outcomes (i.e., RSA and T-CAR) of these youth soccer players. Our hypothesis was that U-19 players would have greater internal training loads than U-15 throughout the preseason phase. As a consequence of these higher training loads placed on older players, U-19 players would exhibit a superior gain in physical performance compared to U-15 players.

\section{Methods}

\section{Participants}

Thirty youth male soccer players were recruited from a professional soccer team competing in the first division of the Brazilian National Championship. The players belonged to one of the following competitive age categories: Under-15 ( $\mathrm{n}=18$; age: $14.7 \pm 0.5$ years, body mass: $59.1 \pm 7.0 \mathrm{~kg}$, body height: $169.1 \pm 7.8 \mathrm{~cm}$, years of systematic practice in soccer: $5.4 \pm 2.3$ years) and Under-19 $(\mathrm{n}=12$; age: $18.9 \pm 0.9$ years, body mass: $67.8 \pm 7.5 \mathrm{~kg}$, body height: $175.1 \pm 7.4 \mathrm{~cm}$, years of experience: $8.6 \pm 1.8$ years). Only outfield players were included in this study. The distribution of the players by a playing position in each competitive age category was as follows: under-15 (3 central defenders, 4 full-backs, 6 midfielders, and 5 attackers) and under-19 (3 central defenders, 2 full-backs, 5 midfielders, and 2 attackers). The inclusion criteria for the study were regular participation in more than $90 \%$ of training sessions during the period of investigation, not suffering from injuries during the same period, and not taking any medication that could alter the outcomes of this study. The study was approved by the local research Ethics
Committee, with participants and their legal guardians (in $<18$ years players) providing written informed assent and consent, respectively, before participation in the study (protocol 1.197.858). The $>18$ years players signed the informed consent form themselves. Participation was voluntary and players could withdraw at any time of the study.

\section{Design and Procedures}

The soccer players were assessed before (pre-training) and after (post-training) an 8-week preseason training period (Table 1). The study period comprised physical training (PT) sessions (interval training, strength, plyometric training, speed), technical (TEC) and tactical (TAC) training, and friendly matches (FM) for both competitive categories. Physical training was defined as a programmed session that was devoted to enabling players to cope with the physical demands of match-play. Sessions focused on player's tactical understanding and/or their technical ability, were defined as TEC/TAC. When the session included both physical and technical activities, it was defined as PT/TEC. Prior to the respective preseason, players had an off-season period of $\sim 1-2$ months. The players completed the following evaluation timetable: 1) medical screening [Week 1: morning], 2) anthropometric assessments [Week 1: Monday and Tuesday afternoon], 3) a repeated-sprint test $(6 \times 40 \mathrm{~m})$ in order to assess both repeated-sprint and change in direction abilities [Weeks 2 and 11: Tuesday afternoon], and 4) Carminatti's Test (T-CAR) aiming to evaluate the intermittent endurance running capacity [Weeks 2 and 11: Thursday afternoon]. The RSA and T-CAR performances were measured the week immediately before and after the beginning and end of the preseason period, respectively. The assessments were performed on a grass field at the training facilities of the professional club. Players were habituated with test procedures as per their usual medical and fitness assessments. A period of 48 hours without training or strenuous exercise was undertaken prior to the physical assessment days to minimize any residual fatigue. During the study period, the internal training load (ITL) of each participant was monitored by means of the session rating of perceived exertion (RPE) method (Foster et al., 2001). The session-RPE method was used as proposed by Foster et al. (2001) to 
quantify the ITL. Thirty minutes after the session, players were asked "How intense was your session?". They were requested to make sure that their RPE referred to the intensity of the whole session. The players were required to give the answer without any contact with each other, to avoid the influence of peer responses. The reported RPE score was then multiplied by the total session duration, in minutes, to indicate the ITL. All players were previously familiarized with the use of the RPE scale.

\section{Repeated Sprint Ability (RSA)}

Before the RSA test, players performed a standardized 10 min warm-up of progressive runs and accelerations that were administered by each age category's physical coach. The RSA test consisted of $6 \times 40 \mathrm{~m}\left(20+20 \mathrm{~m}\right.$ with a $180^{\circ}$ change of direction) sprints separated by $20 \mathrm{~s}$ of passive recovery (Impellizzeri et al., 2008; Rampinini et al., 2007). The players started $0.5 \mathrm{~m}$ behind the start line which was marked by a photocell (Speed Test 6.0 CEFISE®, Nova Odessa, SP, Brazil). Before starting, the players were instructed to run as fast as possible to the end of the $20 \mathrm{~m}$ course, which was marked with 2 cones, then perform a quick change in direction $\left(180^{\circ}\right)$ and run in the direction of the start line. Following each sprint, players decelerated and walked to the starting line in readiness for the subsequent sprint. Five seconds prior to the next sprint, the players assumed the starting position and a $3 \mathrm{~s}$ regressive countdown was provided to commence their sprint. The best (RSAbest) and mean sprint times (RSAmean) were recorded as the performance criteria. The coefficients of variation for RSAmean and RSAbest have been reported to be $0.8 \%$ and $1.3 \%$, respectively, in professional soccer players (Impellizzeri et al., 2008).

\section{Carminatti's Test (T-CAR)}

The test consists of intermittent shuttle runs of $12 \mathrm{~s}$ until volitional exhaustion, performed between 2 lines set at progressive distances, with a $6 \mathrm{~s}$ recovery between each run and a total stage time of $90 \mathrm{~s}$ (Da Silva et al., 2011; Teixeira et al., 2014). The test has a starting velocity of $9 \mathrm{~km} \cdot \mathrm{h}^{-1}$ over a running distance of $30 \mathrm{~m} \mathrm{(15} \mathrm{m} \mathrm{out} \mathrm{and}$ back). The length in a single direction is increased progressively by $1 \mathrm{~m}$ at every level. Each stage consists of 5 repetitions with a $6 \mathrm{~s}$ walking period between 2 lines set $2.5 \mathrm{~m}$ from the starting line (Da Silva et al., 2011; Teixeira et al., 2014). A total of 8-10 players were evaluated simultaneously with the running pace dictated by a pre-recorded audio system (Da Silva et al., 2011; Teixeira et al., 2014). The test ended when participants failed to follow the audio cues on the front line for 2 successive repetitions (using objective criteria applied by observers). The PVT-CAR was calculated from the distance of the final set completed by the player divided by the time to complete the full set of repetitions. In the event of an incomplete set, peak velocity was interpolated using the equation: $\mathrm{PV}=\mathrm{v}+(\mathrm{ns} / 10)^{*} 0.6$, where " $\mathrm{v}$ " was the velocity of the final fully completed stage and "ns" was the number of repetitions completed in the partially completed stage. The peak velocity (PVT-CAR) reached at the end of the test by the athletes was reported as the performance criterion for the TCAR. Reproducibility of the PVT-CAR has been reported previously, with replicate tests within a period of 1 week among 34 youth players aged 10.2-13.0 years. The ICC and measurement error expressed as a coefficient of variation for $\mathrm{PV}_{\mathrm{T}-\mathrm{CAR}}$ were, respectively, 0.89 and $2.30 \%\left(0.3 \mathrm{~km} \cdot \mathrm{h}^{-1}\right)$ (Teixeira et al., 2014).

\section{Statistical Analysis}

The normality of data and homogeneity of variance assumptions were tested using the Shapiro-Wilk and Levene's test, respectively. When assumptions were violated, logtransformations were performed. In order to examine the main training-induced changes in performance measures and differences in the weekly ITL, a two-way repeated measures ANOVA with one between factor (age group: U15 vs. U-19) and one within factor (time: pretraining vs. post-training or over 8-weeks) was used for each dependent variable. When a significant $F$ value was identified, a Bonferroni post hoc test was performed to identify pairwise differences. Student's unpaired $t$ tests were used to compare the total training load and average RPE scores (accumulated over 8-weeks) between age-category groups. Differences between the first and last four weeks for the average total weekly training load and RPE scores within each age group were tested using Student's paired $t$ tests. The level of statistical significance was set at $p<$ 0.05 . These analyses were carried out using SPSS (SPSS 17.0 version, Chicago, Illinois, USA). In addition to the null-hypothesis test, to allow for better interpretation of the results, magnitude- 
based-inference analyses were used to examine the differences between pre-training and posttraining in RSA performance and PVT-CAR. For within- and between-age group comparisons, the chances that the training-induced changes in RSAbest, RSAmean, and PVT-CAR were beneficial/higher (i.e., greater than the smallest worthwhile change, SWC [0.2 multiplied by the between-subject standard deviation]), unclear, or harmful/lower were calculated. Quantitative chances of beneficial/higher or harmful/lower effects were assessed qualitatively as follows: 25 to $75 \%$, possibly; 75 to $95 \%$, likely; 95 to $99 \%$, very likely; and $>99 \%$, almost certainly. If the chances of having higher/beneficial or lower/harmful performances were both $>5 \%$, the true difference was assessed as unclear/similar (Batterham and Hopkins, 2006). In addition, the standardized mean difference or effect size (ES) of changes $(\Delta)$ in RSAbest, RSAmean, and PVT-CAR between the competitive age groups was calculated using the pooled pre-training standard deviation. The criteria to interpret the magnitude of the ES were: $\leq 0.2$ trivial, $>0.2-0.6$ small, $>0.6-1.2$ moderate, $>$ 1.2-2.0 large, and $>2.0-4.0$ very large (Hopkins et al., 2009). All inference-based analyses were conducted using a publicly available spreadsheet (http:// www.sportsci.org/resource/stats/).

\section{Results}

\section{Training Load Profile}

The total weekly training load and average RPE scores during the study period are shown in Figure 1. A significant "age group vs. week" interaction was observed for the total weekly training load (Figure 1a, F = 16.608; $p<$ 0.0001 ) and average RPE score (Figure $1 \mathrm{~b}, \mathrm{~F}=$ 11.055; $p<0.001)$ throughout the 8 weeks of the preseason. Under 19 players presented a greater total training load (Figure 1e) and perceived the training sessions as heavier (Figure 1f) than U-15 players. The training load was lower in week $1(p$ $<0.05)$ in the two competitive age groups. The highest total training load for both the U-15 and U-19 age groups was identified in week 2 (Figure 1a). The average total weekly training load and RPE scores from weeks 1-4 to weeks 5-8 were significantly reduced in the U-15, while remaining unchanged for the U-19 age group (Figures 1c and $1 \mathrm{~d}$, respectively).
Changes in physical performance variables after the preseason

Raw values for all performance variables are presented in Table 2. There was a significant main age group effect for PVT-CAR $(\mathrm{F}=30.300 ; p<$ $0.001)$, RSAbest $(F=30.423 ; p<0.001)$, and RSAmean $(F=42.338 ; p<0.001)$. Compared with the younger group, U-19 players were almost certainly $(100 / 0 / 0)$ better in PVT-CAR (ES: 1.96 and 1.90), RSAbest (ES: 1.78 and 1.48), and RSAmean (ES: 2.87 and 1.73) in both pre-training and post-training moments. After the preseason, all performances (PVT-CAR, RSAbest, and RSAmean) were significantly improved in both groups (all $p<0.001$ for the main time effect). There was no "age group vs. time" interaction for PVT-CAR ( $\mathrm{F}=1.87 ; p=0.18)$ or RSAbest $(\mathrm{F}=1.58 ; p=0.22)$. A trend toward a significant "age group vs. time" interaction was noted for RSAmean $(\mathrm{F}=3.38 ; p=0.08)$.

\section{Magnitude-based analyses: within-age groups comparisons}

Body mass remained unchanged throughout the preseason period with changes being likely trivial in both the U-15 (59.1 \pm 7.0 vs. $59.9 \pm 6.5 \mathrm{~kg}, 6 / 94 / 0)$ and U-19 group (67.8 \pm 7.5 vs. $68.9 \pm 7.0 \mathrm{~kg}, 21 / 79 / 0)$.

Relative changes and qualitative outcomes resulting from the within-group analysis are also reported in Table 2. After the preseason, RSAbest, RSAmean, and PVT-CAR almost certainly improved $(100 / 0 / 0)$, increasing by $4.2 \%$, $5.0 \%$ and $8.0 \%$ in U-15 soccer players, respectively. Large to very large ES for changes was observed. In U-19 soccer players, improvements in RSAbest and RSAmean were very likely beneficial (99/1/0), whereas for the PVT-CAR it was almost certainly beneficial (100/0/0). After the training period, RSAbest, RSAmean, and PVT-CAR improved by $2.7 \%, 3.2 \%$ and $5.0 \%$ in the older age group, respectively. Moderate ES was noticed in RSAbest, RSAmean, and PVT-CAR between the beginning and end of the preseason.

Magnitude-based analyses: between-age groups comparisons for the changes in physical performance

While the "traditional" statistical analyses revealed no differences between age groups for the changes in physical performance variables, analyses of practical significance showed some meaningful differences (Table 3). After the preseason period, changes in RSAbest were possibly 
lower in U-19 than in U-15 players (small ES); while improvements in RSAmean (1/12/87) and PVTCAR $(0 / 18 / 82)$ were likely lower in U-19 soccer players (small ES).

Relationships between the total training load and changes in physical performance

Relationships between the total training load and changes in RSAbest, RSAmean, and PVT-CAR were not significant either in U-15 $(\mathrm{r}=-0.19, p=0.45 ; \mathrm{r}=$ $0.02, p=0.95 ; \mathrm{r}=-0.05, p=0.86)$ or $\mathrm{U}-19$ players $(\mathrm{r}$ $=-0.26, p=0.42 ; \mathrm{r}=-0.27, p=0.39 ; \mathrm{r}=0.52, p=0.09$ ). When data from both groups were pooled, a significant and negative moderate correlation was found between the total training load and changes in RSAmean $(\mathrm{r}=-0.36, p=0.05)$.

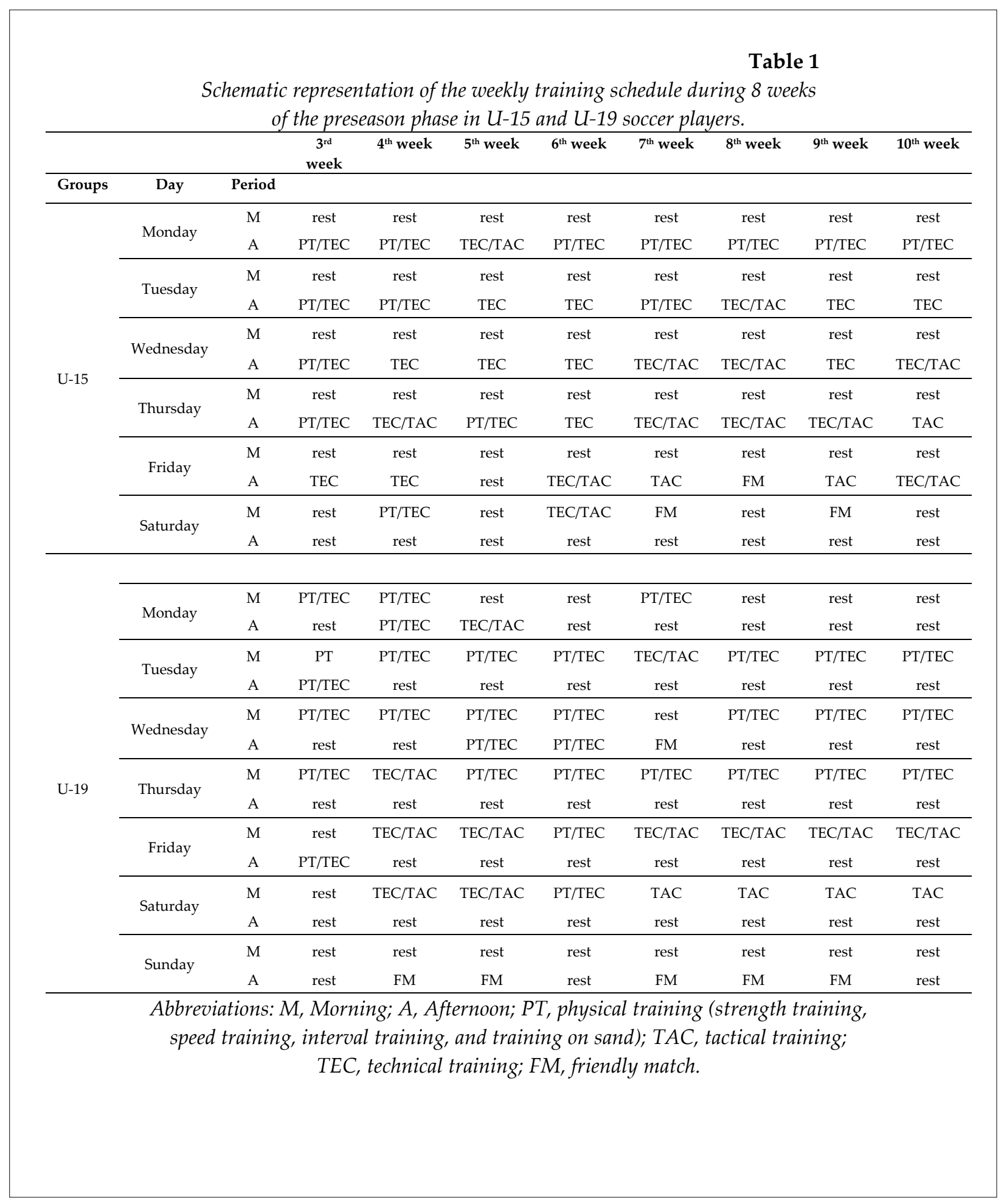


Table 2

Repeated sprint ability (RSA) and T-CAR performance outcomes before (pre) and after (post) an 8 week period of preseason training in under-15 (U-15) and under-19 (U-19) soccer players.

\begin{tabular}{|c|c|c|c|c|c|c|}
\hline \multirow{3}{*}{ Performance } & \multirow{3}{*}{ Groups } & & & \multicolumn{3}{|c|}{ Within-group changes } \\
\hline & & Pre & Post & $\Delta \%$ & $\begin{array}{c}\text { ES } \\
\text { (descriptor) }\end{array}$ & $\begin{array}{l}\text { Qualitative } \\
\text { Inference }\end{array}$ \\
\hline & & Mean \pm SD & Mean \pm SD & & & \\
\hline \multirow[t]{2}{*}{$\mathrm{RSA}_{\text {best }}\left(\mathrm{m} \cdot \mathrm{s}^{-1}\right)$} & U-15 & $5.58 \pm 0.17^{\#}$ & $5.81 \pm 0.16^{\# *}$ & 4.2 (3.1to 5.3) & $\begin{array}{c}1.26 \\
\text { (Large) }\end{array}$ & $100 / 0 / 0$ \\
\hline & U-19 & $5.90 \pm 0.14$ & $6.06 \pm 0.15^{*}$ & 2.7 (1.3 to 4.2$)$ & $\begin{array}{c}1.08 \\
\text { (Moderate) }\end{array}$ & $99 / 1 / 0$ \\
\hline \multirow[t]{2}{*}{$\operatorname{RSAmean}\left(\mathrm{m} \cdot \mathrm{s}^{-1}\right)$} & $\mathrm{U}-15$ & $5.31 \pm 0.09^{\#}$ & $5.58 \pm 0.10^{\# *}$ & $5.0(4.4$ to 5.7$)$ & $\begin{array}{c}2.68 \\
\text { (Very Large) }\end{array}$ & $100 / 0 / 0$ \\
\hline & U-19 & $5.59 \pm 0.17$ & $5.77 \pm 0.11^{*}$ & $3.2(1.3$ to 5.0$)$ & $\begin{array}{c}0.96 \\
\text { (Moderate) }\end{array}$ & $99 / 1 / 0$ \\
\hline \multirow[t]{2}{*}{$\operatorname{PV}$ T-CAR $\left(\mathrm{km} \cdot \mathrm{h}^{-1}\right)$} & U-15 & $15.73 \pm 0.84^{\#}$ & $16.98 \pm 0.68^{\# *}$ & $\begin{array}{c}8.0(5.8 \text { to } \\
10.2)\end{array}$ & $\begin{array}{c}1.39 \\
\text { (Large) }\end{array}$ & $100 / 0 / 0$ \\
\hline & U-19 & $17.45 \pm 0.85$ & $18.34 \pm 0.91^{*}$ & $5.0(1.3$ to 5.0$)$ & $\begin{array}{c}0.94 \\
\text { (Moderate) }\end{array}$ & $100 / 0 / 0$ \\
\hline
\end{tabular}

Columns: \# denotes significant differences between age groups before (pre) and after (post) the preseason period.

Rows: * denotes significant differences within age groups over time $(p<0.05)$.

Table 3

Differences in change observed in repeated sprint ability (RSA) and T-CAR performance outcomes for $U-19$ compared with $U-15$ soccer players.

\begin{tabular}{|c|c|c|c|c|c|}
\hline & & ES $(90 \% \mathrm{CI})$ & Rating & $\begin{array}{c}\text { Percent chance of } \\
\text { higher/trivial/lower } \\
\text { effects }\end{array}$ & $\begin{array}{l}\text { Qualitative } \\
\text { inference }\end{array}$ \\
\hline $\operatorname{RSA} A_{b e s t}\left(\mathrm{~m} \cdot \mathrm{s}^{-1}\right)$ & U-19 vs U-15 & $-0.35(-0.77$ to 0.07$)$ & Small & $2 / 26 / 72$ & Possibly \\
\hline $\operatorname{RSAmean}\left(\mathrm{m} \cdot \mathrm{s}^{-1}\right)$ & U-19 vs U-15 & $-0.53(-1.06$ to -0.01$)$ & Small & $1 / 12 / 87$ & Likely \\
\hline PVT-CAR $\left(\mathrm{km} \cdot \mathrm{h}^{-1}\right)$ & U-19 vs U-15 & $-0.37(-0.70$ to -0.05$)$ & Small & $0 / 18 / 82$ & Likely \\
\hline
\end{tabular}



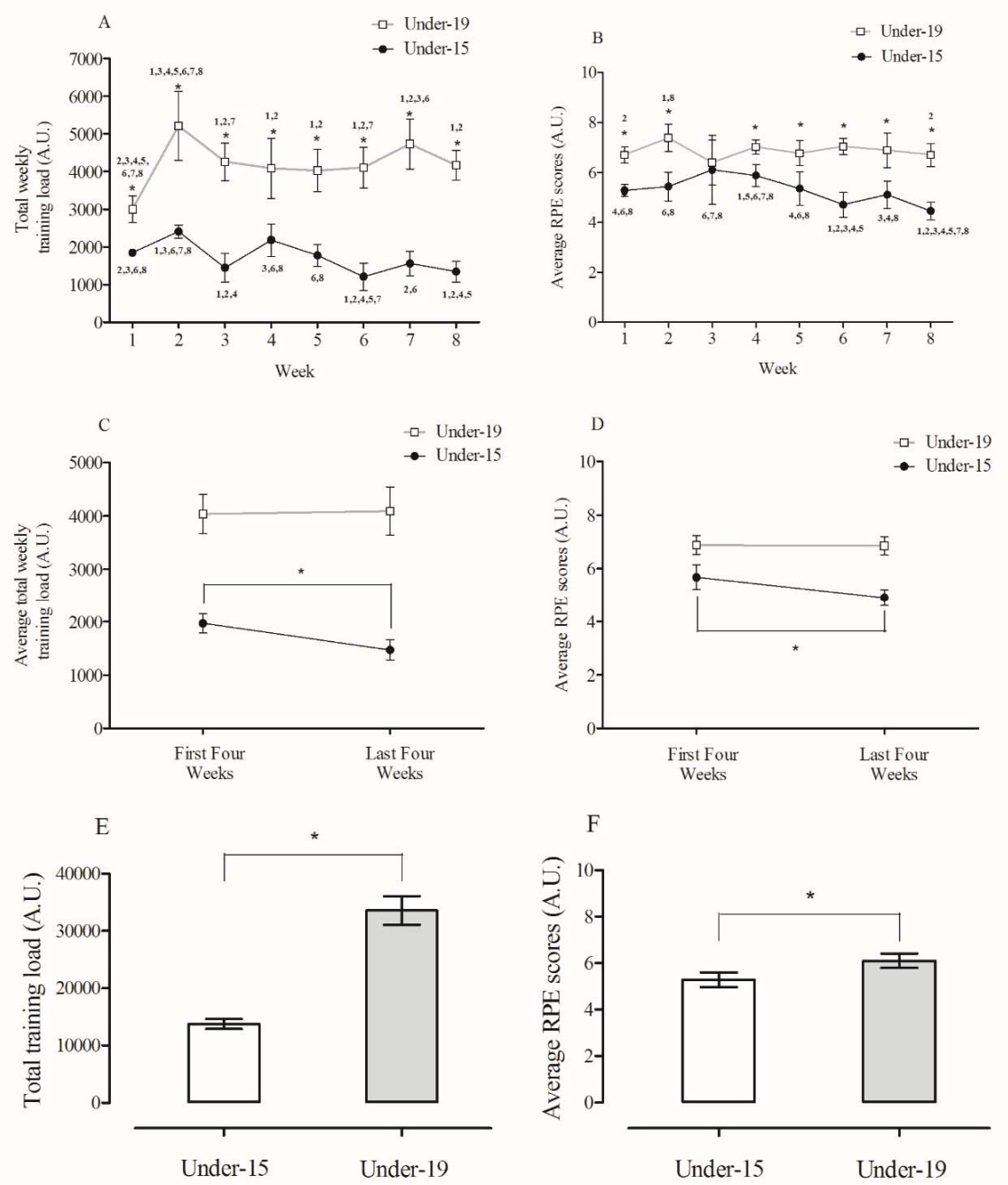

Figure 1

Total weekly training load and average session-rating of perceived exertion (RPE scores) per week ( $A$ and $B$, respectively; upper panel), averaged every four weeks ( $C$ and $D$, respectively; middle panel), and accumulated over 8 weeks ( $E$ and $F$, respectively, lower panel) during the preseason training period in $U-15$ and $U-19$ soccer players.

Abbreviation: A.U., arbitrary units.

$1,2,3,4,5,6,7$ and $8=$ indicates significant difference from week 1, 2, 3, 4, 5, 6, 7 and 8 , respectively.

\# denotes significant differences between the first and the last four weeks $(p<0.001)$;

* denotes significant differences between competitive age groups $(p<0.001)$ 


\section{Discussion}

In the present study, we investigated the internal training loads undertaken by U-15 and U19 soccer players pertaining to the same professional team during 8 weeks of the preseason and the differential responses of the teams regarding the RSA and T-CAR changes. As hypothesized, our results demonstrated that the training loads (volume and intensity) imposed on the soccer players significantly increased from middle adolescence to late adolescence/early adulthood within the same elite development program. Our major finding showed that U-15 soccer players displayed superior gains in RSA and T-CAR performances than U-19 players. Thus, strength and conditioning coaches should be aware that the higher training loads accumulated by U-19 soccer players do not necessarily translate into superior performance adaptations in soccer-specific high-intensity or maximal running tasks. Based on this, our second hypothesis was not confirmed. Furthermore, it was possible to highlight the sensitivity of these field tests (RSA [6 x $20+20 \mathrm{~m}]$ and T-CAR) to track training-induced changes in two distinct and soccer-specific physical abilities (i.e., repeated-sprints ability and intermittent endurance running performance) during a preseason in youth players of different competitive age categories.

Attempts to evaluate the training load by age groups in talented soccer players during adolescence have been poorly described (Gabbett et al., 2014; Wrigley et al., 2012), especially during the preseason phase. In the present study, we demonstrated that U-15 players had a lower total weekly training load than their older counterparts (U-19) during the 8-week preseason. Wrigley et al. (2012) also reported age-related increases in the training load placed upon youth soccer players (U-14, U-16, and U-18) in a short in-season period ( 2 weeks). In contrast to our findings, Wrigley et al. (2012) did not report on significant differences in RPE responses (i.e., training intensity index) across age groups. Overall, our data indicate that from U-15 to U-19 age groups, there was not only progression in the total training load, but also in the activity intensities (Figures 1e and 1f, respectively), in accordance with the long-term development model, respecting the biological traits of the athlete to maximize athletic development while minimizing the risk of overtraining and injury (Ford et al., 2011). A recent study confirmed this fact showing that the higher physiological loading placed on U-17 and U-19 compared to U-15 players was probably related to the use of frequent game-like situations (requiring higher power and speed demands) and less time spent learning technical skills and basic tactical principles during training sessions (Abade et al., 2014).

In general, the emphasis during the preseason is on rebuilding physical performance after detraining that occurs during the off-season (Malone et al., 2015). As a result, high training loads are expected during this phase of the season, emphasizing training methods targeting at physical abilities considered relevant to the sport. In the present study, the highest training load was observed in week 2 for both age groups (U-15 and U-19). For U-15 players, the average total weekly training load and RPE scores within the first four weeks $(1970.35 \pm 178.69$ AU and 5.67 $\pm 0.46 \mathrm{AU})$ were significantly $(p<0.001)$ higher than those obtained in the last four weeks (1472.16 \pm 193.35 AU and $4.90 \pm 0.30 \mathrm{AU}$ ) of the preseason. These results are in agreement with previous studies involving other team sports showing that players undergo the highest training loads within the first weeks of the preseason (Miloski et al., 2015; Oliveira et al., 2013). On the other hand, no significant difference $(p>0.05)$ was observed for the average total weekly training load or RPE score between the first $(4034.39 \pm 368.59$ AU and $6.87 \pm 0.35 \mathrm{AU})$ and the last $(4088.18 \pm 450.06 \mathrm{AU}$ and $6.85 \pm 0.34 \mathrm{AU}$ ) of the four weeks of the preseason for the U-19 age group. This "even" pattern of loading has also been observed in other teams (Nakamura et al., 2016). It remains to be established in the future if "even", "ascending" or "descending" loading patterns during preseasons induce different physical adaptations in soccer players.

Our findings evidenced very likely to almost certainly improvements in both RSAbest and RSAmean (moderate to very large ES) after an 8week typical soccer preseason in players of different age categories. These RSA improvements are in agreement with those reported in previous studies after standardized training regimens, such as repeated sprint and aerobic interval training (Buchheit et al., 2010b; Ferrari Bravo et al., 2008), 
strength training (Buchheit et al., 2010a), and small-sided games (Buchheit et al., 2009). The aforementioned studies reported positive changes in RSAbest (2.1 to $3.5 \%$ ) and RSAmean (2.5 to $3.9 \%$ ) after 4 to 10 weeks of different types of training. The results of the present study are in accordance with these previous studies showing that improvements in RSAmean (3.2 to 5.0\%) tend to be slightly superior to those seen in RSAbest (2.7 to $4.2 \%)$, even though it is known that traininginduced changes in both RSAbest and RSAmean may be attributed to improvements in similar mechanical aspects (e.g., acceleration) (Buchheit et al., 2009).

The changes in repeated-sprint running performance in this study are also likely to be explained by a variety of alterations in the anaerobic metabolism (Spencer et al., 2005) and neuromuscular components (Girard et al., 2011). According to the literature, 8 weeks of the soccer preseason can be sufficient to improve anaerobic ATP production, phosphocreatine resynthesis, and/or blood buffering capacity (Iaia et al., 2015; Spencer et al., 2005). In team sport athletes, maximization of RSA has also been linked to the ability to develop maximal speed (Iaia et al., 2015). For instance, Buchheit et al. (2009) reported moderate correlations $(\mathrm{r}=0.37$ to $0.40 ; p<0.05$ ) between changes $(\Delta)$ in 10-m sprint time and RSA performance outcomes after a training program in handball players. Of interest, tactical and technical training applied in both categories (U-15 and U-19) during the preseason also consisted of small-sided games, which in turn involved performing soccer-specific movements, such as changes of directions, acceleration, and deceleration. Thus, it is likely that the ability to quickly change the direction while running at maximal speeds may also have played a role (Young et al., 2001).

It has been suggested that improvements in intermittent endurance running performance should be emphasized during the early preparatory phases of the training plan since no additional adaptation/gain in the ability to perform intermittent exercise is expected during the in-season period (Krustrup et al., 2003; Oliveira et al., 2013). During the 8-week preseason of this study, T-CAR performance assessed by the PVT-CAR increased by about $8.0 \%$ and $5.0 \%$ for the U-15 and U-19 soccer players, respectively. These relative changes are comparable to those previously reported by Fernandes da Silva et al. (2015) who found an improvement of $7.7 \%$ and $5.4 \%$ in the PVT-CAR after two types of aerobic training (shuttle vs. straight-line runs) in junior soccer players (age: $17.9 \pm 1.0$ years). In addition, standardized differences observed in the current study (ES: 1.39 and 0.94 for U-15 and U-19, respectively) are in fact similar to changes in both Yo-Yo IR1 (ES: +1.2) and 30-15 Intermittent Fitness (ES: +1.1) tests after an 8-week training intervention (Buchheit and Rabbani, 2014), thus highlighting that T-CAR has sensitivity comparable to other traditional field tests to detect training-induced adaptations in the intermittent endurance running capacity of youth soccer players.

Some studies have previously suggested positive associations between the heart rate-based training load and changes in different aerobic fitness measures (Manzi et al., 2009, 2013). In contrast, other studies have shown that the RPEderived training load and subsequent adaptations in aerobic fitness variables are poorly correlated (Akubat et al., 2012; Arcos et al., 2015). Similarly, no significant correlation was obtained in the present study between the RPE-based total training load and anaerobic-aerobic performance adaptations in each age category. However, when data from both age categories were pooled, there was a significant and moderate negative correlation between changes in RSAmean and the RPE-based total training load ( $\mathrm{r}=-0.36 ; p=0.05)$. In agreement with this finding, Arcos et al. (2015) also showed a negative relationship $(\mathrm{r}=-0.54$ and $-0.64 ; p<0.05$ ) between training volume ( $\mathrm{min}$ ) and changes in sprint running performance (i.e., $5 \mathrm{~m}$ and $15 \mathrm{~m}$ times). From a practical point of view, these findings highlight the importance that coaches and strength and conditioning professionals should attach to monitoring the perceived training loads individually in young athletes, especially to avoid/reduce the occurrence of maladaptations in physical performance measures associated with excessive loading during the preseason phase.

In line with this viewpoint, a novel finding of the current study was that the U-15 age group, despite accumulating a lower weekly training load throughout the study period, displayed higher improvements in $\mathrm{PV}_{\mathrm{T}-\mathrm{CAR} \text {, }}$ 
RSAbest, and RSAmean than U-19 soccer players (Table 3). Despite the greater gains in performance during the preseason phase, it should be highlighted that the performance level of younger players in the T-CAR and RSA tests at the end of the preseason was still lower than the performance level reached by their older peers at the beginning of the preseason. This is the first study showing that crucial soccer match-related physical fitness abilities, such as PVT-CAR and RSA, are optimized in different magnitudes of adaptation in well trained U-15 and U-19 players after a regular training process without any external manipulation. In general, our findings are consistent with the results presented by Behringer et al. (2011) in a meta-analysis showing a negative correlation $(r=-0.25 ; p=0.02)$ between the participant's age and the magnitude of change for three physical performance abilities (throwing, jumping, and running) following resistance training programs in physically active children and adolescents. In the same study, Behringer et al. (2011) also showed that youth non-athletes presented greater enhancements in physical abilities than highly trained athletes. This indicates that younger boys during adolescence, especially around the peak height velocity (Meylan et al., 2014; Philippaerts et al., 2006), may experience higher gains in physical and motor performance than their older counterparts. From this perspective, our findings indicate that there might be a ceiling effect of functional adaptations in older players, requiring higher training loads to obtain lesser gains in physical performance in the RSA and T-CAR.

\section{Practical Implications}

The current study showed that total weekly RPE-based training loads varying between 1000 and $2000 \mathrm{AU}$ for U-15 and $\approx 4000 \mathrm{AU}$ for U19 soccer players can provide a sufficient stimulus to induce positive gains in RSA and intermittent endurance running performance during a preseason period. Coaches and strength and conditioning professionals should be aware that PVT-CAR, RSAbest and RSAmean are trainable, but to different magnitudes of adaptations, in U-15 and U-19 soccer players. Such findings should assist coaches to optimize the timing of training programs designed to improve these two crucial soccer-specific physical abilities, taking into account the differences in age-related responsiveness. In addition, our findings demonstrated the sensitivity of these two field tests (T-CAR and RSA [6 $\times 20+20 \mathrm{~m}]$ ) to identify important training-induced adaptations in soccer players of different competitive age categories.

\section{Conclusions}

In conclusion, our findings indicate that U-19 soccer players had a higher training volume and perceived the training sessions as heavier than U-15 players. Consequently, older players accumulated a greater total training load throughout the preseason phase than U-15 soccer players. Changes in PVT-CAR and RSA performance outcomes were very likely to almost certainly beneficial after the preseason period in both competitive age categories, having U-15 players achieved relative gains possibly to likely greater in high-intensity and maximal running performances than U-19 soccer players.

\section{Acknowledgements}

We would like to thank for the patience and cooperation of the athletes and coaches during the execution of this study and the Conselho Nacional de Desenvolvimento Científico e Tecnológico (CNPq) for financial support.

\section{References}

Abade EA, Gonçalves BV, Leite NM, Sampaio JE. Time-motion and physiological profile of football training sessions performed by under-15, under-17 and under-19 elite Portuguese players. Int J Sports Physiol Perform, 2014; 9(3): 463-470

Akubat I, Patel E, Barrett S, Abt G. Methods of monitoring the training and match load and their relationship to changes in fitness in professional youth soccer players. J Sports Sci, 2012; 30(14): 1473-1480

Arcos AL, Martínez-Santos R, Yanci J, Mendiguchia J, Méndez-Villanueva A. Negative Associations between Perceived Training Load, Volume and Changes in Physical Fitness in Professional Soccer Players. J 
Sports Sci Med, 2015; 14(2): 394-401

Batterham AM, Hopkins WG. Making meaningful inferences about magnitudes. Int J Sports Physiol Perform, 2006; 1: 50-57

Behringer M, Vom Heede A, Matthews M, Mester J. Effects of strength training on motor performance skills in children and adolescents: a meta-analysis. Pediatr Exerc Sci, 2011; 23(2): 186-206

Buchheit M, Laursen PB, Kuhnle J, Ruch D, Renaud C, Ahmaidi S. Game-based training in young elite handball players. Int J Sports Med, 2009; 30(4): 251-258

Buchheit M, Mendez-Villanueva A. Effects of age, maturity and body dimensions on match running performance in highly trained under-15 soccer players. J Sports Sci, 2014; 32(13): 1271-1278

Buchheit M, Mendez-Villanueva A, Delhomel G, Brughelli M, Ahmaidi S. Improving repeated sprint ability in young elite soccer players: repeated shuttle sprints vs. explosive strength training. J Strength Cond Res, 2010a; 24(10): 2715-2722

Buchheit M, Mendez-Villanueva A, Quod M, Quesnel T, Ahmaidi S. Improving acceleration and repeated sprint ability in well-trained adolescent handball players: speed versus sprint interval training. Int $J$ Sports Physiol Perform, 2010b; 5(2): 152-164

Buchheit M, Mendez-Villanueva A, Simpson BM, Bourdon PC. Match running performance and fitness in youth soccer. Int J Sports Med, 2010c; 31(11): 818-825

Buchheit M, Millet GP, Parisy A, Pourchez S, Laursen PB, Ahmaidi S. Supramaximal training and postexercise parasympathetic reactivation in adolescents. Med Sci Sports Exerc, 2008; 40(2): 362-371

Buchheit M, Rabbani A. The 30-15 intermittent fitness test versus the yo-yo intermittent recovery test level 1: relationship and sensitivity to training. Int J Sports Physiol Perform, 2014; 9(3): 522-524

Coutinho D, Gonçalves B, Figueira B, Abade E, Marcelino R, Sampaio J. Typical weekly workload of under 15, under 17, and under 19 elite Portuguese football players. J Sports Sci, 2015; 33(12): 1229-1237

Czuba M, Maszczyk A, Gerasimuk D, Roczniok R, Fidos-Czuba O, Zając A, Gołaś A, Mostowik A, Langfort $\mathrm{J}$. The effects of hypobaric hypoxia on erythropoiesis, maximal oxygen uptake and energy cost of exercise under normoxia in elite biathletes. J Sports Sci Medic, 2014; 13(4): 912-920

Da Silva JF, Castagna C, Teixeira AS, Carminatti LJ, Guglielmo LGA. The peak velocity derived from the Carminatti Test is related to physical match performance in young soccer players. J Sports Sci, 2016; 34(24): 2238-2245

Da Silva JF, Guglielmo LGA, Carminatti LJ, De Oliveira FR, Dittrich N, Paton CD. Validity and reliability of a new field test (Carminatti's test) for soccer players compared with laboratory-based measures. J Sports Sci, 2011; 29(15): 1621-1628

Da Silva JF, Nakamura FY, Carminatti LJ, Dittrich N, Cetolin T, Guglielmo LGA. The effect of two generic aerobic interval training methods on laboratory and field test performance in soccer players. J Strength Cond Res, 2015; 29(6): 1666-1672

Ferrari Bravo D, Impellizzeri FM, Rampinini E, Castagna C, Bishop D, Wisloff U. Sprint vs. interval training in football. Int J Sports Med, 2008; 29(8): 668-674

Ford P, De Ste Croix M, Lloyd R, Meyers R, Moosavi M, Oliver J, Till K, Williams C. The long-term athlete development model: physiological evidence and application. J Sports Sci, 2011; 29(4): 389-402

Foster C, Florhaug JA, Franklin J, Gottschall L, Hrovatin LA, Parker S, Doleshal P, Dodge C. A new approach to monitoring exercise training. J Strength Cond Res, 2001; 15(1): 109-115

Gabbett TJ, Whyte DG, Hartwig TB, Wescombe H, Naughton, GA. The relationship between workloads, physical performance, injury and illness in adolescent male football players. Sports Med, 2014; 44(7): 989-1003

Gastin PB, Bennett G, Cook J. Biological maturity influences running performance in junior Australian 
football. J Sci Med, 2013; 16(2): 140-145

Girard O, Mendez-Villanueva A, Bishop D. Repeated-sprint ability - part I: factors contributing to fatigue. Sports Med, 2011; 41(8): 673-694

Hopkins WG, Marshall SW, Batterham AM, Hanin J. Progressive statistics for studies in sports medicine and exercise science. Med Sci Sports Exerc, 2009; 41(1): 3-13

Iaia FM, Fiorenza M, Perri E, Alberti G, Millet GP, Bangsbo J. The Effect of Two Speed Endurance Training Regimes on Performance of Soccer Players. PLoS One, 2015; 10(9): e0138096

Impellizzeri FM, Rampinini E, Castagna C, Bishop D, Ferrari Bravo D, Tibaudi A, Wisloff U. Validity of a repeated-sprint test for football. Int J Sports Med, 2008; 29(11): 899-905

Krustrup P, Mohr M, Amstrup T, Rysgaard T, Johansen J, Steensberg A, Pedersen PK, Bangsbo J. The yo-yo intermittent recovery test: physiological response, reliability, and validity. Med Sci Sports Exerc, 2003; 35(4): 697-705

Lloyd RS, Oliver JL. The Youth Physical Development Model: A New Approach to Long-Term Athletic Development. Strenght Cond. J, 2012; 34(3): 61-72

Lloyd RS, Oliver JL, Faigenbaum AD, Myer GD, De Ste Croix MBA. Chronological age vs. biological maturation: implications for exercise programming in youth. J Strength Cond Res, 2014; 28(5): 1454-1464

Malone JJ, Di Michele R, Morgans R, Burgess D, Morton JP, Drust B. Seasonal training-load quantification in elite English premier league soccer players. Int J Sports Physiol Perform, 2015; 10(4): 489-497

Manzi V, Bovenzi A, Franco Impellizzeri M, Carminati I, Castagna C. Individual Training-Load and AerobicFitness Variables in Premiership Soccer Players During the Precompetitive Season. J. Strength Cond. Res, 2013; 27(3): 631-636

Manzi V, Iellamo F, Impellizzeri F, D'Ottavio S, Castagna C. Relation between individualized training impulses and performance in distance runners. Med Sci Sports Exerc, 2009; 41(11): 2090-2096

Mendez-Villanueva A, Buchheit M, Simpson B, Bourdon PC. Match play intensity distribution in youth soccer. Int J Sports Med, 2013; 34(2): 101-110

Meylan CMP, Cronin JB, Oliver JL, Hopkins WG, Contreras B. The effect of maturation on adaptations to strength training and detraining in 11-15-year-olds. Scand J Med Sci Sports, 2014; 24(3): e156-64

Miloski B, De Freitas VH, Nakamura FY, De A. Nogueira FC, Bara-Filho MG. Seasonal training load distribution of professional futsal players. J Strength Cond Res, 2015; 30(6): 1525-1533

Nakamura FY, Pereira LA, Rabelo FN, Ramirez-Campillo R, Loturco I. Faster Futsal Players Perceive Higher Training Loads and Present Greater Decreases in Sprinting Speed During the Preseason. J Strength Cond Res, 2016; 30(6): 1553-1562

Noon MR, James RS, Clarke ND, Akubat I, Thake CD. Perceptions of well-being and physical performance in English elite youth footballers across a season. J Sports Sci, 2015; 33(20): 2106-2115

Oliveira RS, Leicht AS, Bishop D, Barbero-Álvarez JC, Nakamura FY. Seasonal changes in physical performance and heart rate variability in high level futsal players. Int J Sports Med, 2013; 34(5): 424-430

Oliver JL, Lloyd RS, Whitney A. Monitoring of in-season neuromuscular and perceptual fatigue in youth rugby players. Eur J Sport Sci, 2015; 15(6): 514-522

Philippaerts RM, Vaeyens R, Janssens M, Van Renterghem B, Matthys D, Craen R, Bourgois J, Vrijens J, Beunen G, Malina RM. The relationship between peak height velocity and physical performance in youth soccer players. J Sports Sci, 2006; 24(3): 221-230

Rabelo FN, Pasquarelli BN, Gonçalves B, Matzenbacher F, Campos FAD, Sampaio J, Nakamura, FY. Monitoring the Intended and Perceived Training Load of a Professional Futsal Team Over 45 Weeks: A Case Study. J Strength Cond Res, 2016; 30(1): 134-140

Rampinini E, Bishop D, Marcora SM, Ferrari Bravo D, Sassi R, Impellizzeri FM. Validity of simple field tests 
as indicators of match-related physical performance in top-level professional soccer players. Int J Sports Med, 2007; 28(3): 228-235

Roescher CR, Elferink-Gemser MT, Huijgen BCH, Visscher C. Soccer endurance development in professionals. Int J Sports Med, 2010; 31(3): 174-179

Spencer M, Bishop D, Dawson B, Goodman C. Physiological and metabolic responses of repeated-sprint activities: specific to field-based team sports. Sport Med, 2005; 35(12): 1025-1044

Teixeira AS, Da Silva JF, Carminatti LJ, Dittrich N, Castagna C, Guglielmo LGA. Reliability and validity of the Carminatti's test for aerobic fitness in youth soccer. J Strength Cond Res, 2014; 28(11): 3264-3273

Vaeyens R, Lenoir M, Williams AM, Philippaerts RM. Talent identificaton and development programmes in sport. Sports Med, 2008; 38(9): 703-714

Vaeyens R, Malina RM, Janssens M, Van Renterghem B, Bourgois J, Vrijens J, Philippaerts RM. A multidisciplinary selection model for youth soccer: the Ghent Youth Soccer Project. Br J Sports Med, 2006; 40(11): 928-934

Valente-dos-Santos J, Coelho-e-Silva MJ, Martins RA, Figueiredo AJ, Cyrino ES, Sherar LB, Vaeyens R, Huijgen BCH, Elferink-Gemser MT, Malina RM. Modelling developmental changes in repeated-sprint ability by chronological and skeletal ages in young soccer players. Int J Sports Med, 2012; 33(10): 773-780

Wrigley R, Drust B, Stratton G, Scott M, Gregson W. Quantification of the typical weekly in-season training load in elite junior soccer players. J Sports Sci, 2012; 30(15): 1573-1580

Young WB, McDowell MH, Scarlett BJ. Specificity of sprint and agility training methods. J Strength Cond Res, 2001; 15(3): 315-319

\section{Corresponding author:}

\section{Anderson Santiago Teixeira}

Federal University of Santa Catarina

Sports Center - Physical Effort Laboratory

Campus Universitário - Trindade

CEP: 88040-900 Florianópolis (SC) Brazil

Phone: +05548 3721-9924

E-mail: anderson.santeixeira@gmail.com 